at once that a woman, to whom he was called at the first commencement of labour, had a small male type of pelvis, and be sent her up to me at the hospital forthwith. I operated at once; she made a recovery, as aseptic abdowinal cases do. She has frequently been up to the ward with her child; she has not been again pregnant, and so may be one of the women alluded to above, who have but one opportunity.

Renal Obstruction and Suppression of Urine.

If we may include renal among abdominal cases, then suppression, or threatened suppression, of urine will form the chief indication for immediate operation when we can find evidence that the cause is obstructive. An $x$-ray examination may show calculi on both sides, or even slight distension of the kidney and pelvis. In the absence of an $x$-ray examination, one must rely upon the history of the case and upen palpation. But, if there is any suspicion, in face of suppression, that the cause is an obstructive one which will not yield to medical treatment, early nephrotomy should be done. If done early, one may do it on one side first, and wait before cutting into the opposite kidney.

\section{ON THE USES OF CATGUT IN SURG ERY: \\ WITH SPECIAL REFERENCE TO IODINE CATGUT AND IODINE-FORMALIN CATGUT.}

By C. YELVERTON PEARSON, M.D., F.R.C.S., PROPESSOR OF SURGERY, ONITERSITY COLLEGE, CORK.

Considrring the advances that have been made of recent years in the preparation of catgut by improved and simplified methods, it is strange that such diversity of opinion should still exist amongst surgeons of repute regarding its safety and general applicability for surgical purposes. At the present time operating surgeons adopt one of the following attitudes regarding its use

1. There may be some who never employ it.

2. There are those who employ it to a very limited extent, and chiefly in infected wounds.

3. There are others who, eitber owing to some unfarourable experience or to the reports of tetanus and other disasters in the practice of other surgeons, use it with a feeling of uncertainty and anxiety.

4. Finally, there are those who use it freely in all cases where such a material is suitable.

As a striking example of such diversity in practice I may mention that during a recent visit to the United States I found that in the Mayos' Clinic at Rochester, Minn., iodine catgut was extensively employed for ligatures and buried sutures, to the complete exclusion of other materials, save where some more resisting substance is necessary-for example, chromicized gut is used for hernia operations and for the inner row of gastro-intestinal sutares, the external row consisting of iron.dyed Irish linen. The iodine catgut used is sterilized by Willard Bartlett's method, ${ }^{1}$ which consists in heating it in liquid petrolatum previous to its being placed in a 1 per cent. solation of iodine crystals in Columbian spirits. This preliminary sterilization is somewhat troublesome and quite unnecessary for the preparation of iodine catgut, as I shall presently point out. The use of catgut so prepared has, however, given the greatest satisfaction; it has been used by the brothers Mayo and their assistants in over 5,000 operations with most satisfactory results, and has been adopted by Murphy, Deaver, and others.

In the surgical clinic of Johns Hopkins Hospital, on the other hand, I found that catgat is used to only a very limited extent, iron.dyed silk, freshly boiled, being extensively employed for ligatures and buried satures. Dr. Finny informed me that he avoided the use of catgut as far as possible, owing to the tronble to which it frequently gave rise, notwithstanding its most carefal preparation by various methods; the process which still found most favour there being the difficult and cumbersome cumol method.

It is my belief that so long as surgeons continue to employ a simple aseptic catgut, no matter what the pro cess of sterilization employed, so long must they expect to have trouble and anxiety with their operation wounds.
I have elsewhere ${ }^{2}$ taken considerable pains to point out the objections to the use of plaini sterile catgut, and insisted on having it impregnated with some reliable antiseptic. This is one of those exceptional instances in which antisepsis as opposed to simple asepsis possesses a distinct advantage.

Ewald found that catgut has the power of exciting sappuration, even when it is perfectly sterile; this property he attributed to an unknown toxic substance. The experiments of Klemm led him to believe that though catgnt may be sterile, it affords a suitable soil for the cultivation of micro-organisms. Apart, however, from infection, as I have pointed out, "it appears that catgut sometimes possesses chemotactic properties which lead to the formation in the tissues of sterile pus," 8 or, at least, a fluid which differs only from true pus in the absence of pyogenic micro-organisms.

During the past few years several cases of postoperative tetanus have been reported, and in some at least of the cases catgut has been held responsible, and, I believe, not unjustly, though I do not know what kind of catgut was used. What I desire specially to point out is that, even assuming the catgut to be sterile but devoid of antiseptic properties, $I$ think it not only possible, but highly probable, that if placed in a favourable situation in the peritoneal cavity, owing to its chemical properties it might attract to it micro organisms from the intestinal canal such as $B$. coli, $B$. enteritidis, or possibly some organism which, though not identical with the $B$. tetani, may be capable of developing a tetanic toxin.

Now, if catgut be not alone sterile, but also impregnated with some germicidal substance which protects it from infection, this could not occur. It is not enough that the gut is incorporated with one or another antiseptic; the antiseptic itself must possess certain qualities to be ideal. It must be strongly antiseptic, preferably germicidal, so that it not only protects the gut from infection bat also sterilizes its track; it must be able to penetrate the gat throughout, without injuring its physical properties; be non-toxic and non-irritant; not interfere with timely absorption and must make the catgat antiseptic throughout its entire active life

So far, the substance which has given for this purpose most general satisfaction is iodine, which, though powerfully germicidal as well as antiseptic, does not, when brought into contact with the deeper tissues in solutions of moderate strength, seem to exert any injurious influencet Nor have I ever seen or heard of a case of iodism from the free use of catgut impregnated with it, which shows that it is not rapidly absorbed.

\section{Iodized Catgat.}

The method of preparing iodized catgut which first gained it popnlarity was that of Claudius, which con. sisted in immersing the gut in a 1 per cent. watery solution of iodine and potassium iodide for at least eight days. It was said that the catgut might be kept in this solution for a considerable time without deteriorating in quality. This I soon found to be a fallaey, and at the same time satisfied myself that loss of tensile strength was not due to the evaporation of the iodine, as suggested by Mr. A. S. Barling, ${ }^{4}$ but to the chemical action of the water on the fibrous tissue. This softening by simple maceration will be found to take place in fibrous tissue when it is immersed for any considerable time in distilled water, in the absence of all putrefactive changes and even in the presence of some antiseptics, but will not occur in the presence of various hardening agents-and more especially alcohol, which has the further advantage that it does not produce brittleness.

It was owing to the recognition of this action of watery solutions of iodine on catgat that I was led to use different methods for its preservation, three of which are described in my work on Modern Surgical Technique as being satisfactory-namely:

(1) The addition to the aqueous iodine solution of an equad quantity of alcohol; (2) the addition to it of an equal quantity of 5 per cent. phenol solution, which, by combining with the iodine, removes most of the colour, but gives an antiseptic catgut that will keep admirably; (3) transferring the catgut to catgut that will keep admirably; (3) transferring the catgut to a spirituous preserving solution of mercuric biniodide, having
previously washed it with phenol solution to remove excess of previously washed it with
lodine from its surface. 
I continued to employ the first of the above methods for some months, but as I could not see the advantage of soaking the catgut for eight days in a watery solation before adding any alcohol, and as I was satisfied that it softened the gut, this method of preparation quickly gave way to simple immersion of the catgut in equal parts of Claudius's solution and duty free spirit, in which I found it would keep indefinitely without loss of strength or pliability.

I may point out that I had adopted this method of preparation previous to the appearance of the article by Dr. J. Scott Riddell in the British Medical Journal, April 6th, 1907, on "Iodine spirit catgut," or to my knowledge of its having been suggested by Salkindsohn. Personally I do not consider that the "iodine spirit catgat" prepared by his method contains a sufficient amount of iodine, as the solution, which is made by adding one part of tincture of iodine to fifteen parts of proof (50 per cent.) alcohol, contains only 0.15 per cent. iodine, the strength of the tincture being 2.5 per cent. Moreover the use of iodine tincture and proof spirit is quite unnecessary, and the fact that both are subject to duty adds considerably to the cost of preparation. Anyone who requires only a small amount of iodine catgut for private operations may prepare it with a mixture of equal parts of tincture of iodine and distilled water. This gives 1.25 per cent. iodine to 45 per cent. alcohol.

For practical purposes a mixture of equal parts of Claudius's 1 per cent. watery solution and daty-free spirit will answer, and, as I have mentioned, was for a considerable time used in the hospitals to which I am attached; but this 0.5 per cent. solution does not yield the darkbrown or nearly black catgut which is by Claualus and others considered desirable, and for hospital purposes I now advise the following:

Make a 10 per cent. iodine stock solution by rubbing up the requisite quantity of iodine with twice its weight of potassium or sodium iodide and sufficient distilled water.

Take of this 10 per cent. iodine stock

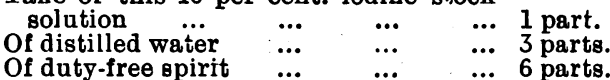

This gives a 1 per cent. solution of iodine in 54 per cent. alcohol (approximately).

The catgut is placed in this for at least eight days. It may then be placed in jars, and just covered with a solu. tion containing one half the amount of iodine. It will keep in this indefinitely, and is only taken out a short time before it is required for use, so as to allow some of the alcohol to evaporate.

Those who prefer a dry iodine catgut, as advised by Moschcowitz, can readily obtain it by placing it after preparation for a short time in full strength 90 per cent. alcoholic solution, removing, placing on a sterile dish under a glass bell-jar, and allowing it to dry spontaneously. I cannot see that dry catgut possesses any advantages, as that prepared in the solution recommended is easily manipulated, and does not swell during use from the absorption of moisture from the tissues, as is certain to happen with the dry, which is also said to become brittle, and must lose some of its iodine by evaporation.

\section{Iodine.Formalin Catgut.}

When I learnt that Boeckman had shown that the action of formalin on catgut makes its surface vaterproof, so that boiling water does not penetrate the gut, and that, as a consequence, the interior of the ligature is only exposed to a dry heat of $212^{\circ} \mathrm{F}$., which does not sterilize, ${ }^{5}$ I came to the conclusion that it was not safe to use formalin catgut prepared in the ordinary way. It occurred to me that it might be possible first of all to iodize the catgut and then to harden its outer layers by immersion in formalin. I tried this over two years ago, and the experiment proved so successful that I have since then been using "iodine.formalin" catgut wherever delayed absorption was desirable, with most satisfactory results.

The method of preparation is as follows:

1. The gut is prepared with an alcoholic solution of iodine in the manner already described.

2. At the end of eight days it is taken from the iodine solution with sterile forceps, placed in a sterile glass jar, and washed with either a weak solution of carbolic acid or with running sterile water to remove the alcohol and iodine from the outer layers, so as to permit more freedom for the action of the formalin.

3 . It is placed in a 3 per cent. formalin solution for from twenty-four to forts-eight hours according to the thickness of the gut.

4. It is washed in running water for a few hours to remove the formalin.

5. It is now placed in 50 per cent. alcohol containing 0.5 per cent. iodine and 5 per cent. glycerine, this latter rendering the gut more pliable.

In this solution it may be kept indefinitely, being taken from it only shortly before required for ase to allow the alcohol to evaporate.

When preparing either "iodine" or "iodine-formalin" gut, the finer qualities are wound on my fluted glass spools, which permit free access of the fluid. The coarser qualities of gut are cut into lengths of about $2 \frac{1}{2} \mathrm{ft}$.; each of these is made into a small coil about $1 \frac{1}{2}$ in. in diameter, one free end being twisted a few times around the coil to keep it from opening. The coils are then strung on a thread and suspended in the solution during preparation, but are allowed to rest on the bottom when placed in the final preserving solution.

Catgut prepared in this way possesses the combined advantages of iodine and formalin gat without any of the disadvantages of formalin gut prepared in the ordinary manner.

In the British Medical Journal, October 2nd, 1909, Mr. F. J. Steward, of Gay's Hospital, published some notes on "formalin.iodine" catzut without apparently being aware of the fact that the use of catgut prepared first with formalin and then with iodine, by the method of Claudius, was adrocated in 1904 by W. J. Stone. ${ }^{6}$ Now, is it not probable that as formalin renders the outer layers of catgut impermeable to boiling water, so also will it render them impermeable to any iodine solution, and that, consequently, resistant pathogenic micro-organisms included in the original manufacture of the raw gut may escape destruction?

The Uses of Catgut.

Owing to its being an absorbable substance, catgut should be employed for surgical purposes whenever and wherever an absorbable material will answer the required purpose, and a little reflection will show that this applies to almost all cases in which ligatures or buried sutures are used.

In infected wounds or in parts that are liable to become infected an absorbable ligature or suture is the only safe material to use, unless a non-absorbent substance, such as metallic wire, be employed, which cannot itself become infected. For example, if a suture such as silk, which though presumably sterile at the time of its being placed in the tissues, subsequently becomes infected with pyogenic organisms, it will keep up continued suppuration in the part so long as it is permitted to remain there. I have come across some cases operated on by others for movable kidney in which silk had been used for the nephropexy, and in which, oring to its becoming infected, suppurating sinuses persisted for over twelve months, but were quickly cured by removal of the infected sutures.

When the desired result can be attained in a short time it is preferable that the ligature should undergo rapid absorption, as further retention of the material in the tissues can serve no useful purpose. Hence in such cases plain iodine catgut should be selected. This applies to all ligatures for blood vessels and pedicles, for the suturing of soft parts to obliterate dead spaces where there is not much tension, and for securing the temporary retention of a drainage tube, as in cholecystotomy, etc.

On the other hand, where the action of the suture is desired for a considerable period, it is necessary to use some material that will resist absorption. This indication can be completely falfilled by the use of iodine-formalin catgut, which will remain unabsorbed and retain its strength for from two to several weeks, according to its thickness. My own experience and observations agree with those of Mr. Steward, and show that a suture of No. 3 iodine-formalin catgut will remain unabsorbed in the abdominal parietes without much loss of tensile strength for over four weeks, while No. 1 will last, under similar conditions, for at least two weeks. We are therefore justified in using this material for the suturing ef nerves, tendons, fasciae, muscular strata, the care of 
hernia, and even for uniting fragments of bone, more especially fractured patella.

For operations on the stomach and intestines, I think it is advisable that an absorbable suture should be used next the lumen, as no one can foretell the ultimate fate of nonabsorbable thread which is partially in contact with the digestive juices and partially etmbedded in the coats of the stomach or intestines. Unhardened catgut is unsuitable, as it is quickly dissolved, so that most surgeons employ either formalin or chromicized catgut. The formaliniodine catgut is particularly suitable and reliable for the purpose. For the outer layer of intestinal suture, which soon becomes embedded in plastic lymph, it is customary to use a non-absorbable material, and it seems a matter of indifference whether silk, linen thread, or the celluloidcoated thread of Pagenstecher is selected.

Where a suture is required that will undergo slow absorption, chromicized catgut is usually recommended. For reasons that I have already explained, I would not feel safe in using a plain chromicized gut, and it has been shown by Mr. Charles H. Christal 7 and others that catgut prepared with chromic acid, and even subsequently with sulphurous acid, is not necessarily sterile. This difficulty can be got over by its subsequent treatment with a solution of thymol in spirit, as suggested by $\mathrm{Mr}$. Christal, or by treating sulpho-chromic catgut with iodine, as employed in the Leeds Infirmary, and probably many other places. I have had such complete satisfrction with the combined formalin-iodine catgut, that I have had no temptation to employ any form of chromicized gut since I commenced using the former. The sizes of catgut used may vary with the wish of the operator. For ordinary ligatures I use iodine catgut No. .0 for the smaller vessels, No. 1 for the larger, and No. 3 or 4 for pedicles. For suturing the abdominal parietes, I select No. 1 iodine-formalin gut for the inner or transperitoneal suture, and No. 3 for the aponeurotic or muscular layers. It is not necessary, as a rule, to use coarser sizes, and when additional strength is required a double suture will answer. To have too many sizes only leads to confusion.

\section{General Conclusions.}

1. The use of simple aseptic catgut is unsafe, no matter how it is prepared.

2. Iodine catgut may be safely used in all cases in which a quickly absorbable suture or ligature will answer.

3. The use of iodine-formalin catgut is indicated when slower absorption of the suture material is desired, and if prepared in the manner indicated it may be used in all those instances in which it has been customary to use chromicized or sulpho-chromic catgut.

4. The operating surgeon should be personally respon. sible for the manner in which any catgut he uses is prepared.

1 Journ. Amer. Med.Assoc., A pril 21st,1906. 2 Pearson, Modern Surgical Technique. ${ }^{3} \mathrm{Op}$. cit. ${ }^{4}$ BRITISH MEDICAL JoURNAL, August 27th, 1904. SManual of Operative Surgery, Binney, 1906. 6 Medical Record, November 12th, 1904. 7 Lancet, July 10th, 1909.

THE

\section{REMOVAL OF FOREIGN BODIES FROM THE OESOPHAGUS BY MEANS OF BRUNING'S} DIRECT OESOPHAGOSCOPE.

BY J. GAY FRĖNCH, M.S.LoND., F.R.C.S.ENG., SURGEON IN CHARGE OF THE EAR, NOSE, AND THROAT DEPARTMENT, GREAT NORTHERN CENTRAL HOSPITAL; ASSISTANT SURGEON TO

THE following two cases, which recently occurred in two consecutive weeks in the ear, nose, and throat department of the Great Northern Central Hospital, show the importance of direct oesophagoscopy both for the purposes of examination and the location and removal of foreign bodies in the oesophagus. This method is a perfectly simple one in the ordinary adult patient, and the various manipulations can be easily carried out after a little practice.

CASE I.

A woman, aged 42 came to the out-patient department on Tuesday, June 8th, 1909. She stated that three days previously she had swallowed a large piece of unmasti- cated mutton, and that it had "stuck in her chest." She came up to the hospital on the same evening and was seen by one of the house-surgeons, who passed an oesophageal bougie but could detect nothing abnormal. She had been able to swallow fluids only since her visit, and stated that the act of swallowing caused her considerable pain.

Unfortunately I did not have an $x$-ray photograph taken of the case, as I was under the impression that the dysphagia probably resulted from some slight abrasion produced by the passage of the oesophageal bougie, and also owing to the fact that the woman expressly stated that she was quite sure that the trouble was due to a piece of meat and not a bone. I decided to pass Bruning's oesophagoscope and make an examination. The patient's pharynx and epiglottis were anaesthetized with a ten per cent solution of cocaine; she was placed on a low stool, the body being kept straight and the head thrown far back and steadied in this position by an assistant. Braning's oesophagoscope was then passed down as far as it would go and the extension tube introduced. To my surprise I found at a distance of $34 \mathrm{~cm}$. from the upper incisor teeth, in the region where the oesophagus is constricted in its passage through the diaphragm, a piece of bone fixed transversely. At the positions where the ends of the bone were embedded in the oesophagus, the walls looked sloughy and the whole of the surrounding area was markedly congested and oedematous. The bone was firmly fixed in this position, and I found that it would be impossible to remove it with forceps without doing extensive damage to the oesophageal mucous membrane. I therefore passed a sharp hook, manipulating it behind and under the bone, which was steadied against the end of the oesophagoscope tube, and cut it across; the pieces then came away quite easily from the wall.

The woman was kept in for a few days under observation and then discharged. She came up a fortnight afterwards, seying that she had experienced no further discomfort since the removal, and that she could now swallow her food normally.

Case II.

A female child aged 1 year and 10 months was brought by her mother to the out-patient department on Tuesday, June 15th, 1909, with the following history: Four days

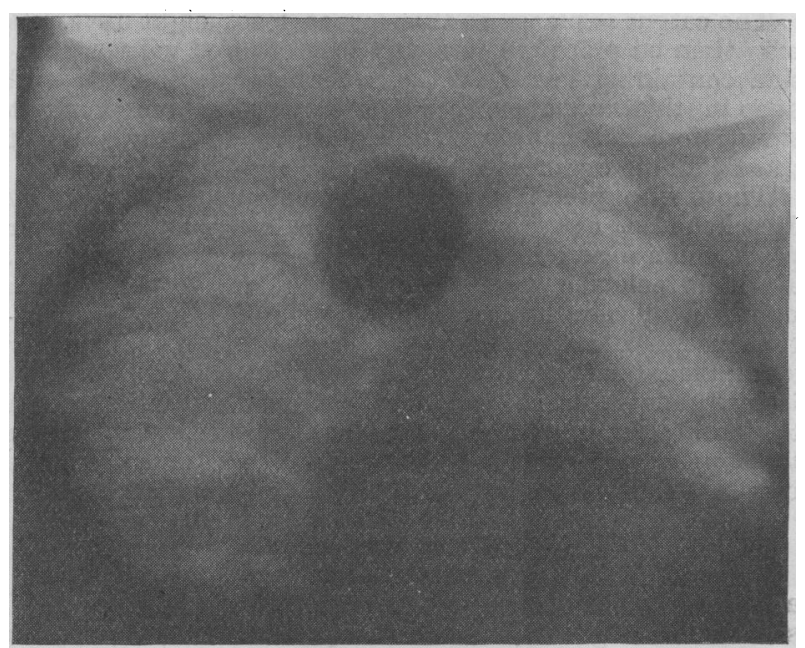

Fig. 1.-Radiograph taken from the front.

previously the child was left in the care of an elder brother, who gave her a button to play with, which she put into her mouth, when it "slipped down." The mother took her on the same evening to an infirmary, where an exami. nation was made but nothing found. She was told to bring the child up again, should any untoward symptom arise. The mother states that she kept the infant as quiet as possible; but found that she was unable to swallow anything except a little fiuid. On Monday evening, on finding that it was impossible to get any fluid down at all, the mother brought the child to the Great Northern Central Hospital, where she was seen by one of the house-surgeons, who examined her in the $x$-ray room with a screen. He found a definite shadow of the button in the intrathoracic portion of the oesophagus, and attempted to remove this 